Research Article

\title{
Research on Multiattribute Comprehensive Evaluation of Intelligent Judicial Decision System
}

\author{
Gang Zhao, Jifa Wang, and Huibin Shi (iD \\ School of Management, Shenyang University of Technology, No. 111, Shenliao West Road, \\ Economic \& Technological Development Zone, Shenyang 110870, China \\ Correspondence should be addressed to Huibin Shi; hhbbs@live.cn
}

Received 7 August 2021; Accepted 23 August 2021; Published 3 September 2021

Academic Editor: Daqing Gong

Copyright (c) 2021 Gang Zhao et al. This is an open access article distributed under the Creative Commons Attribution License, which permits unrestricted use, distribution, and reproduction in any medium, provided the original work is properly cited.

When dealing with cases, judges must consult a large number of relevant materials and carefully consider before they can write the final judgment. So, we want to use intelligent systems to assist the judicial system in handling cases. The essence of the system is automatic text classification. The system can predict the judgment result according to the previous prediction and can also provide support for judicial judgment and individual litigation. Because the evaluation of intelligent judicial decision-making system has the characteristics of complexity and fuzziness, we establish a comprehensive evaluation model of intelligent judicial decisionmaking system with subjective and objective combination by introducing the TOPSIS model. In the experiment, firstly, we use nine multiattribute comprehensive evaluation index systems such as acquisition cost and use cost to grade the indexes. Secondly, AHP and entropy weight methods are used to calculate the subjective weight and objective weight of the index, respectively; the combined weight of the index is determined according to the expert forced scoring method, the attribute measurement function of a single index is constructed according to the classification of the index, the comprehensive attribute measurement is calculated, and the comprehensive evaluation grade is judged according to the attribute identification standard. Finally, taking the intelligent judicial decision-making system as the research object, combined with the system report and expert score, this paper makes a multiattribute comprehensive evaluation and analysis of the intelligent judicial decision-making system and analyzes the results. The final experimental results show that the evaluation results of the model are reasonable and consistent with the actual situation, which verifies the adaptability of the combined weighted attribute recognition model in the multiattribute comprehensive evaluation of intelligent judicial judgment system. This result provides ideas and theoretical follow-up work for the intelligent judgment of judicial cases and has certain significance for the development of the field of judicial judgment.

\section{Introduction}

Intelligent court is a form of people's court organization, construction, operation, and management that relies on modern artificial intelligence, pays attention to people's justice, fairness, and justice, adheres to judicial law, system reform, and technological change, supports judicial trial, litigation service, and judicial management, and realizes all-round online processing, full process disclosure according to law, and all-round intelligent services [1-4]. This provides convenience for the court to deal with cases, but it is not very popular at present, so it is necessary to provide new ideas for intelligent justice.

Judicial personnel inevitably have to engage in a lot of repetitive work in their career. Statistics from Liu et al. show that, in the first half of 2017, the total number of cases handled by the Chinese mainland courts was 14 million 586 thousand, and 8 million 887 thousand of them were eventually terminated, with a $60.9 \%$ rate and $60.9 \%$ cases. Compared with the same period in 2016, the number of cases accepted by national courts increased by $11.2 \%$, of which $14.8 \%$ were taken over, $9.88 \%$ were closed, and $13.54 \%$ were not closed. There are about 120000 judges nationwide, with 121.4 cases per capita and 74 cases per capita. It can be seen that the workload of judges is huge [5]. In fact, a judge has to consult a large number of relevant materials to conclude a case and write the final judgment after careful consideration. This is a great challenge for judges, both professional ability and physical quality. If the judicial assistance system is 
applied to deal with and solve these cases, it can save most human and material resources, greatly improve its work efficiency, and reduce the pressure [6]. This paper proposes that the judicial assistance system is just such an attempt. The essence of the system is the automatic classification of texts. The system can predict the judgment results (support or rejection) of private lending cases according to previous precedents, so as to provide support for judicial decisions and individual litigation.

On May 11, 2017, Qiang Zhou, President of the Supreme People's Court of China, stressed at the fourth national court informatization work conference: we should make overall plans and comprehensively grasp the overall layout of the construction of smart courts [7]. The construction of smart courts should aim at promoting the modernization of the judicial system and judicial capacity and improving the level of justice for the people and justice. We should deeply realize that informatization is the operation carrier of the organization, management, and construction of people's courts. Smart courts are a form of people's court work based on informatization and actively promote the intelligent operation of people's court work in the smart court system healthy development. We should accurately grasp the networking, sunshine, and intelligence characteristics of smart courts, take whether they meet the "all business, all process, and all-round" as the basic standard and main basis for evaluating smart courts, and create green justice under the supervision of the government [8].

\section{Literature View}

Since the 1970s, decision support system (DSS) was first proposed in the management decision system by Scott Morton and Peter g. W. keen [9]. It has attracted extensive attention in academic circles because DSS is a technology integration system, integrating computer technology, information technology, artificial intelligence, management science, decision science, psychology, organization theory, and other disciplines and technologies. With the continuous development of these disciplines, especially, the great breakthrough of computer technology and information technology, it can be predicted that DSS, as a new interdisciplinary science, will make a breakthrough with their rapid development. At present, the decision support system has achieved good results in the fields of medicine, finance, enterprise risk assessment, law, and so on [10-13]. For example, the IFPs' system developed by American Express is a dialogue financial planning system to assist managers in planning and decision-making. The MAS system developed by Huazhong University of Science and Technology is used to optimize the allocation and transportation of various resources in the railway department [14]: in February 2017, the "Shanghai intelligent criminal auxiliary case processing system" of Shanghai high people's court, etc. [15].

Text classification is to automatically classify and mark text sets (or other entities or objects) according to a certain classification system or standard. According to a set of labeled training documents, it finds the relationship model between document features and document categories and then uses the learned relationship model to judge the category of new documents. Text classification has gradually changed from knowledge-based method to statistics and machine learning method. In 1981, Professor Hanqing Hou discussed and expounded the application of computer in text classification. Since then, some text classification systems have been studied and produced in China, including the automatic classification system of Tsinghua University and the research of text classifier based on neural network algorithm $[16,17]$. At the same time, extensive research and implementation have been carried out in different classification algorithms. Xiaoli Li and Zhongzhi Shi of the Institute of computing of the Chinese Academy of Sciences have applied conceptual reasoning network to text classification, with a recall rate of $94.2 \%$ and an accuracy rate of $99.4 \%$ [18]. Yan Fan of University of Science and Technology of China and others proposed a hypertext coordination classifier based on KNN, Bayes, and document similarity research. The accuracy is close to $80 \%$. Its characteristic is that it properly considers the structured information in HTML text [19]. Xuanjing Huang and Lide Wu of Fudan University and Fujitsu Research Center studied the text classification of independent languages, took the mutual information of vocabulary and categories as the scoring function, and considered single classification and multiclassification, and the best recall rate was $88.87 \%$ [20]. Qian Diao and Yongcheng Wang of Shanghai Jiaotong University combined word weight and classification algorithm to classify. In the closed test experiment based on VSM, the classification accuracy reached $97 \%[21,22]$.

\subsection{Combined Weighting Attribute Recognition Model}

2.1.1. Combination Weighting. The subjective weight calculation method relies too much on the subjective cognition of the evaluator. The objective weight calculation method emphasizes too much on the attribute of the data itself and the accuracy of the data. The two calculation methods are combined to calculate the index combination weight, and the weight result is more balanced and reasonable. The specific calculation process is as follows.

Determination of index weight by improved analytic hierarchy process (IAHP): construct judgment matrix, hierarchical single ranking, and consistency test. According to the scale and according to the information and data, combined with the scoring of experts in the cold chain logistics industry, the judgment matrix at all levels is obtained. The maximum eigenvalue of each judgment matrix is calculated by the sum product method $\lambda$ Max and weight coefficient $W$. At the same time, the consistency index CI and random consistency ratio $\mathrm{Cr}$ were calculated, and the consistency was judged.

In this study, the importance of directly related elements at each level can be compared by constructing a judgment matrix and calculated.

The weight value of each design element in each level is calculated, and the comparison element $X_{i j}$ in the judgment matrix adopts the 1-9 scale proposed [23]. 
2.1.2. Weight Calculation. According to the judgment matrix, the geometric average method is used to calculate the weight of design elements of the criterion layer and the subcriterion layer. The approximate operation steps are as follows.

(1) Calculate the scale product for each row:

$$
M_{i}=\prod_{j=1}^{m} b_{i j}, \quad(i=1,2, \ldots, 3)
$$

where $b_{i j}$ is the demand index in row $I$ and column $J$ and $M$ is the evaluation index.

(2) Judge the geometric average of the scale product of each line:

$$
a_{i}=\sqrt[m]{M_{i}}, \quad i(1,2, \ldots, 3) .
$$

(3) Calculate relative weights:

$$
W_{i}=\frac{a_{i}}{\sum_{i=1}^{m} a_{i}}
$$

(4) Calculate the maximum characteristic root:

$$
\lambda_{\max }=\frac{1}{n} \sum_{i=1}^{n} \frac{B_{W i}}{W_{i}},
$$

where $B_{W i}$ is the $i$ th component of vector $B_{W}$ and $n$ is the order

(5) Result consistency test:

$$
\mathrm{CI}=\frac{\lambda_{\max }-n}{n-1},
$$

where $n$ is the order of the evaluation scale of the judgment matrix:

$$
\mathrm{CR}=\frac{\mathrm{CI}}{\mathrm{RI}}
$$

where RI is the average random consistency index, and each order has corresponding values, as shown in Table 1 , and $\mathrm{Cr}$ is the consistency ratio. When $\mathrm{Cr} \leq 0.1$, it indicates that the consistency test is passed. When CR $>0.1$, it means that the consistency inspection has not passed, and the judgment matrix needs to be checked, corrected and calculated, and analyzed again.

(1) Determination of index weight by entropy weight method:

(1) Quantify each index in the same degree, and calculate the proportion $p_{i j}$ of the index value of the $i$ th scheme under the $j$ index:

$$
p_{i j}=\frac{x_{i j}}{\sum_{i=1}^{m} x_{i j}} .
$$

(2) Calculate the entropy $e_{j}$ of the $j$ th index:
TABle 1: Average random consistency index.

\begin{tabular}{lccccccccc}
\hline$n$ & 1 & 2 & 3 & 4 & 5 & 6 & 7 & 8 & 9 \\
\hline $\mathrm{RI}$ & 0 & 0 & 0.52 & 0.89 & 1.12 & 1.26 & 1.36 & 1.41 & 1.46
\end{tabular}

$$
e_{j}=-k \sum_{i=1}^{m} p_{i j} \ln p_{i j}
$$

where $k>0, \ln$ is the natural logarithm, and $e_{j} \geq 0$. If $x_{i j}$, for a given $j$, is all equal, then

$$
p_{i j}=\frac{x_{i j}}{\sum_{i=1}^{m} x_{i j}}=\frac{1}{m} \text {. }
$$

At this time, $e_{j}$ takes the maximum value as

$$
e_{j}=-k \sum_{i=1}^{m} \frac{1}{m} \ln \frac{1}{m}=k \ln m .
$$

If we set $k=(1 / \ln m)$, then we have $0 \leq e_{j} \leq 1$.

(3) Calculate the difference coefficient $g_{j}$ of index J: for a given $j$, the smaller the difference of $x_{i j}$, the greater the difference of $e_{j}$. When $x_{i j}$ are all equal, $e_{j}=e_{\max }=1$. At this time, indicator $x_{j}$ has no effect on scheme comparison. When the difference between the index values of each scheme is greater, $e_{j}$ is the smaller, and the index plays a greater role in the comparison of schemes.

(4) Define weights:

$$
a_{j}=\frac{g_{i}}{\sum_{j=1}^{n} g_{i}} .
$$

(2) The integrated weight formula:

(1) TOPSIS model construction: for the objectivity of the evaluation results, according to the index weight $w_{i}$, create normalized analysis matrix C:

$$
C=\left|y_{i j}\right|_{m \times n}=\left|w_{i j} \times x_{i j}\right|_{m \times n} .
$$

(2) Determine the positive and negative ideal values: positive ideal solution $Z^{+}$and negative ideal solution $Z^{-}$are the maximum and minimum values of the $i$ th index in $j$ years. The specific formula is

$$
\begin{aligned}
& z^{+}=\max \left\{y_{i j}\right\}, \\
& z^{-}=\min \{y i j\} .
\end{aligned}
$$

(3) Calculate the distance: the European distance is selected to calculate the distance from the safety evaluation index of China's titanium resources to the positive and negative ideal. $D^{+}$is the distance between the $i$ th index and $Z^{+}$, and $D^{-}$is the distance between the $i$ th index and $Z^{-}$. The specific formula is 


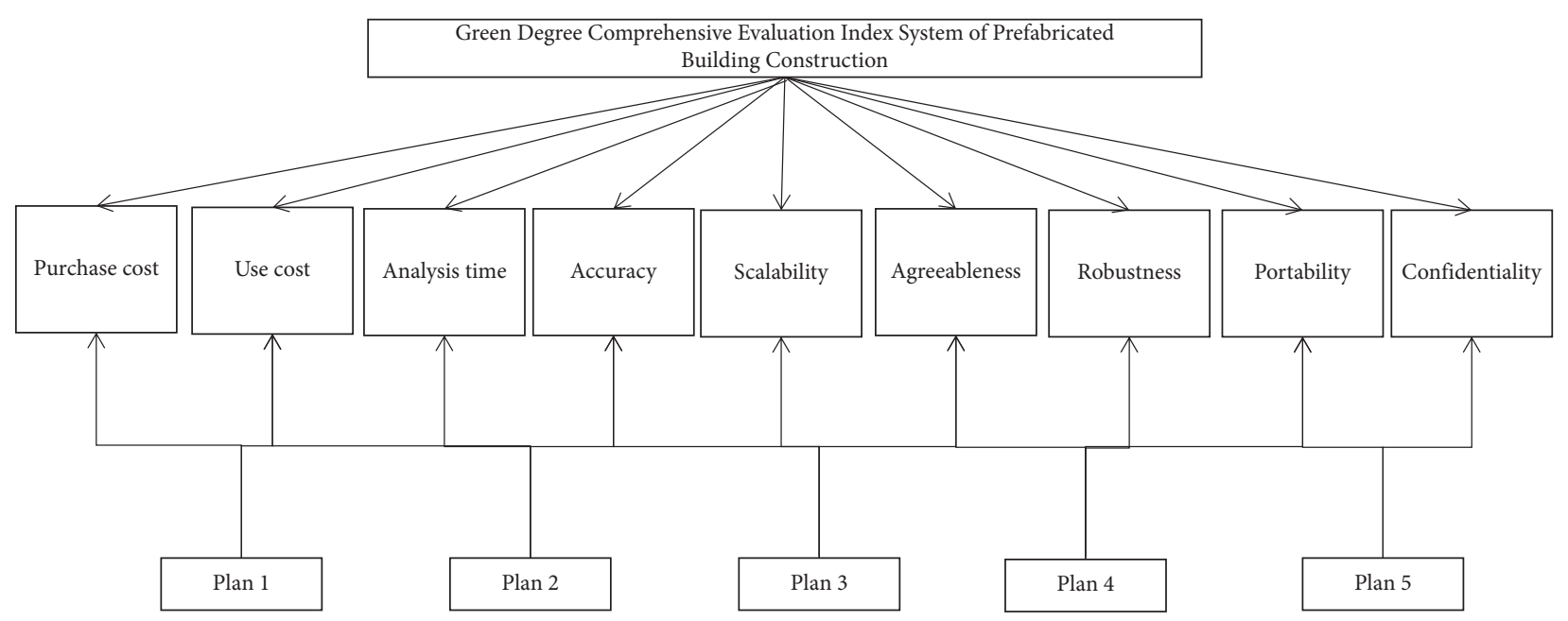

Figure 1: Green evaluation index system of prefabricated buildings.

$$
\begin{aligned}
& D^{+}=\sqrt{\sum_{i=1}^{m}\left(Z_{i}^{+}-y_{i j}\right)^{2}}, \\
& D^{-}=\sqrt{\sum_{i=1}^{m}\left(Z_{i}^{-}-y_{i j}\right)^{2}} .
\end{aligned}
$$

(4) Calculate the comprehensive evaluation index: $C_{j}$ is the comprehensive safety evaluation index of China's Titanium Resources in the $j$ th year. The greater the value, the higher the safety degree. The calculation formula is

$$
C_{j}=\frac{D_{j}^{-}}{D_{j}^{+}+D_{j}^{-}}, \quad C_{j} \in[0,1] .
$$

2.2. Build a Comprehensive Evaluation Index System. In order to verify the applicability of the multiattribute comprehensive evaluation method of the intelligent judicial decision system proposed in this paper, relevant calculations are carried out with the scheme selection of the intelligent judicial decision system as the research background. The intelligent judicial judgment system pays attention to the combination of theory and practice, adopts various methods such as example, group interaction, and simulated judgment, focuses on the combination of classic cases and legal provisions, and carries out reference decision-making output with the help of computer language development environment software. In the process of practice, be familiar with the text data analysis process and technical methods and strengthen the practical application ability in relevant fields. Software system can play an important role in text data mining and has the ability to deeply learn and master the technology of text data analysis. Quantitative indicators are determined by specific data, and qualitative indicators are determined by experts. Because software system factors are interrelated, the selection of indicators should follow the principles of comprehensiveness, science, and measurability.
This paper constructs the evaluation index system from nine aspects: purchase cost, use cost, analysis time, accuracy, scalability, agreeableness, robustness, portability, and confidentiality. Based on the life cycle theory of software development, use, and maintenance, this paper constructs the multiattribute comprehensive evaluation index system of the intelligent judicial decision system .

The evaluation of the intelligent judicial judgment system has received the comprehensive influence of many factors, and the specific index selection is shown in Figure 1.

\section{Model Application}

\subsection{Index Weight Calculation}

(1) Improve the analytic hierarchy process to determine the subjective weight of indicators, and organize experts to rank the importance of criteria level and target level indicators, respectively. According to the above operation flow of geometric average method, combined with the survey results of the judgment matrix, the relative weight of design elements at each level is calculated through scale product and relative weight, and the ranking results of relative importance given by experts are shown in Table 2. Calculate the index weight of each index layer according to formulas (7) (11).

(2) Calculate the maximum characteristic root. In order to ensure the consistency of the research object's thinking and the compatibility of the judgment matrix during the evaluation, the results of the consistency test of the judgment matrix are assisted by calculating the maximum eigenvalue. The results show that the $C R$ values are less than 0.1, indicating that they have passed the consistency test, as shown in Table 3:

$$
\lambda_{\max }=\frac{1}{n} \sum_{i=1}^{1} \frac{B_{W i}}{W_{i}} .
$$

In equation (2), $B_{W i}$ is the $i$ th component of vector $B_{W}$ and $n$ is the order 


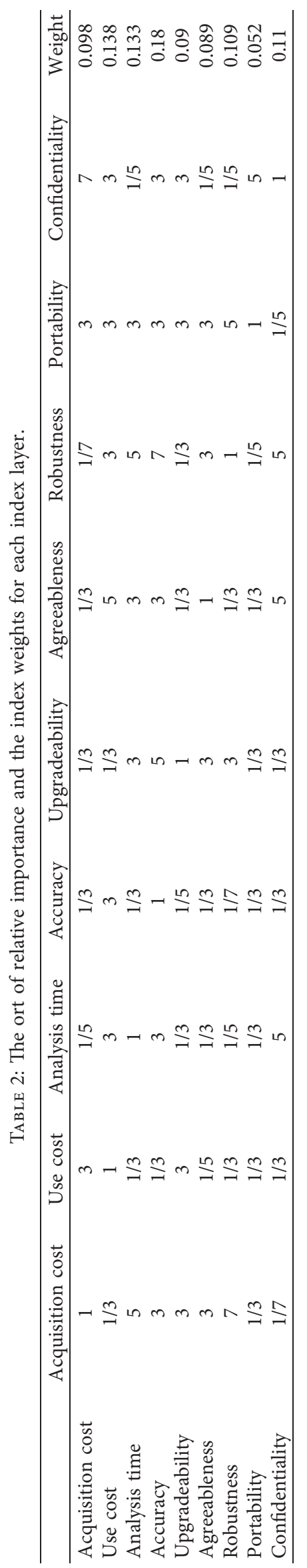


TABLE 3: The consistency test results of the matrix by calculating the maximum eigenvalues.

\begin{tabular}{|c|c|c|c|c|c|c|c|c|c|}
\hline$B_{W i}$ & 16.414 & 17.433 & 15.008 & 16.04 & 15.838 & 14.44 & 13.47 & 16.648 & 17.488 \\
\hline$W_{i}$ & 1.602 & 2.402 & 1.992 & 2.891 & 1.43 & 1.292 & 1.474 & 0.868 & 1.929 \\
\hline$\left(B_{w i} / W_{i}\right)$ & $\begin{array}{c}1.138 \\
10.025\end{array}$ & 0.806 & 0.837 & 0.616 & 1.231 & 1.242 & 1.015 & 2.131 & 1.007 \\
\hline
\end{tabular}

(3) Result consistency test:

$$
\mathrm{CI}=\frac{\lambda_{\max }-n}{n-1}
$$

The result is $(10.024-9) /(9-1)=0.128$.

In equation (3), $n$ is the order of the evaluation scale of the judgment matrix:

$$
\mathrm{CR}=\frac{\mathrm{CI}}{\mathrm{RI}}
$$

RI $(9)=1.46, C R=0.128 / 1.46=0.088$, less than 0.1 , passed the consistency test

(4) The objective weight of the index is calculated by the entropy weight method.

Combined with the index values given in Table 4, take the intermediate value of each index classification to form the initial decision matrix. After normalization and entropy calculation, the index weight is obtained. The specific results are $(0.0382$, $0.0394,0.0396,0.0398,0.0397,0.0382,0.0388$, $0.0388,0.0344,0.0398,0.0268,0.0376,0.0359$, $0.0376,0.0398,0.0390,0.0396,0.0396,0.0390$, $0.0395,0.0398,0.0394,0.0397,0.0398$, and 0.0397):

$$
\begin{array}{llllll}
0.135 & 0.04 & 0.133 & 0.009 & 0.459 & 0.03 \\
0.088 & 0.076 & 0.029 & & &
\end{array}
$$

(5) The combination weight is used to calculate the index combination weight. According to formulas (12) (13), the combination weight of indicators is determined based on the principle of minimum entropy, and the calculation results are (0.0441, $0.0367,0.0246,0.0302,0.0202,0.0658,0.0810,0.0543$, 0.0343, 0.0450, 0.0511, 0.0513, 0.0424, 0.0311, 0.0271, 0.0374, 0.0273, 0.0241, 0.0397, 0.0446, 0.0199, 0.0120, $0.0349,0.0309,0.0511$, and 0.0309).

The combination weight is $w_{i}$.

3.2. Ideal Deconstruction. According to the above operation program, the initial evaluation matrix is normalized in combination with the weight of analytic hierarchy process to obtain the weight normalization matrix. Through the calculation formula of positive and negative ideal solutions, it is obtained that the positive ideal solution $=(3.8,4.8,60,98.5$, $4,95,88,55,9)$ and the negative ideal solution $=(5.2,5.7,82$, $90,2,80,68,42,8)$, which are calculated by Euclidean algorithm and relative closeness. The results shown in Table 5 are obtained. Among them, the priority of the scheme is judged according to the CI value. The larger the value, the higher the priority of the scheme. Therefore, the best scheme 2 can be obtained.

3.3. Comprehensive Attribute Measurement. According to Table 5 and equation (16), the comprehensive evaluation index of the intelligent judicial decision system to be evaluated can be obtained. The comprehensive evaluation order of the intelligent judicial decision system is scheme $5>$ scheme $2>$ scheme $4>$ scheme $1>$ scheme 3 , that is, scheme 5 . On the basis of comprehensively considering the balance between experts' previous experience and practical schemes, the distance order between the scheme and the optimal ideal solution scheme is given, which shows that the comprehensive evaluation result is reasonable and reliable.

3.4. Model Discussion. This paper establishes a comprehensive evaluation model based on combined weight attribute recognition and applies the model to the evaluation of the intelligent judicial decision system. Compared with other models, the model has the significant advantages of simple calculation, intuitive, and strong operability. In the calculation process, it does not need to set too many parameters, is easy to master and apply, is scientific and practical, and has a certain popularization value. According to the evaluation results, the main influencing factors of the evaluation of the intelligent judicial judgment system are purchase cost, use cost, analysis time, accuracy, upgradeability, agreeableness, robustness, portability, and confidentiality. According to the ranking of experts, the order of importance is accuracy, use cost, analysis time, confidentiality, robustness, purchase cost, upgradeability, agreeableness, and portability.

According to the entropy weight method, the order of importance is upgradeability, purchase cost, analysis time, robustness, portability, use cost, agreeableness, confidentiality, and accuracy. It should be pointed out that the accuracy rate is the most important factor in expert judgment and the least important factor in objective ranking. The reason is that the accuracy rate of all schemes in objective factors has reached a very high level, so the weight is relatively low.

In this decision-making process, the subjective weight and objective weight are given the same importance. In the evaluation of the specific intelligent judicial judgment system, different degrees of importance can be used to adjust according to a certain proportion. 
TABLE 4: The objective weight of the index is calculated by the entropy weight method.

\begin{tabular}{|c|c|c|c|c|c|c|c|c|c|}
\hline & Acquisition cost & Use cost & Analysis time & Accuracy & Upgradeability & Agreeableness & Robustness & Portability & Confidentiality \\
\hline Plan 1 & 4 & 5.7 & 78 & 98 & 3.5 & 80 & 75 & 48 & 9 \\
\hline Plan 2 & 4.3 & 5.5 & 60 & 95 & 2 & 90 & 68 & 45 & 8 \\
\hline Plan 3 & 5 & 5.6 & 65 & 98.5 & 3.9 & 85 & 88 & 50 & 9 \\
\hline Plan 4 & 3.8 & 5 & 80 & 90 & 4 & 90 & 70 & 55 & 8 \\
\hline Plan 5 & 5.2 & 4.8 & 82 & 97 & 3.7 & 95 & 83 & 42 & 9 \\
\hline
\end{tabular}

Table 5: Positive and negative ideal solutions and their relative proximity.

\begin{tabular}{lcccc}
\hline Plan & $\mathrm{s}^{+}$ & $\mathrm{s}^{-}$ & $\mathrm{c}$ & Rank \\
\hline Plan 1 & 0.121830839 & 0.014767394 & 0.108108238 & 4 \\
Plan 2 & 0.005385504 & 0.188155141 & 0.972173781 & 2 \\
Plan 3 & 0.382013286 & 0.005944692 & 0.015323032 & 5 \\
Plan 4 & 0.000153537 & 0.004936119 & 0.969833488 & 3 \\
Plan 5 & 0.000135665 & 0.010458829 & 0.987194785 & 1 \\
\hline
\end{tabular}

\section{Conclusion}

(1) In order to improve the scientificity of the weight calculation results, the analytic hierarchy process and entropy weight method are used to calculate the subjective weight and objective weight of the index, respectively, and the index combination weight is determined based on the expert scoring method. The combination of the two makes the weight results more balanced and can improve the reliability of the evaluation results.

(2) Considering the complexity and practical characteristics of intelligent judicial judgment system evaluation, TOPSIS (technology for order preference by similarity to an ideal solution) method is introduced. The research results show that scheme 5 is at the best level, closest to the positive ideal solution, and farthest from the negative ideal solution. The evaluation results are in line with the reality, indicating that the model has certain adaptability in the evaluation of the intelligent judicial judgment system. It can provide ideas and theoretical guidance for the evaluation work. Finally, in order to further improve the operability of the project, reasonable suggestions are put forward.

(3) Because there are many factors affecting the evaluation of the intelligent judicial judgment system, but the theoretical research is still insufficient, and the evaluation model has not been unified. In the future research, it is necessary to build a more perfect index evaluation system and comprehensively consider the actual situation of the project in order to obtain satisfactory evaluation results.

\section{Data Availability}

The data used to support the findings of the study can be obtained from the corresponding author upon request.

\section{Conflicts of Interest}

The authors declare that they have no conflicts of interest.

\section{Acknowledgments}

This work was financially supported by Liaoning Province Planning Office of Philosophy and Social Science (L19BGL028).

\section{References}

[1] Y. Q. Liu and W. Yueyue, "Informatization and intellectualization: discrimination in the judicial context," China Applied Law, vol. 5, no. 2, pp. 15-30, 2021.

[2] Y. Lu and Y. J. Zheng, "Application of artificial intelligence in the field of judicial adjudication," Journal of Zhongzhou University, vol. 38, no. 3, pp. 41-46, 2021.

[3] S. Q. Zhang and N. Kang, "Practice and orientation of artificial intelligence in judicial adjudication," Journal of Shandong Judges Training College, vol. 37, no. 3, pp. 91-109, 2021.

[4] X. Liu, "More cases and fewer people' and the reform of judge post system," Research on Political Law, vol. 7, no. 1, pp. 86-111, 2017.

[5] Y. N. Shuai, "Realistic possibility and necessary limit of artificial intelligence assisted judicial adjudication," Journal of Shandong University, vol. 53, no. 4, pp. 101-110, 2020.

[6] Q. Zhou, "Scientific and technological innovation drives the construction of smart courts, judicial services guarantee scientific and technological innovation," People's Court News, vol. 19 , no. 5 , p. 2, 2021.

[7] A. L. Awaga, W. Xu, L. Liu, and Y. Zhang, "Evolutionary game of green manufacturing mode of enterprises under the influence of government reward and punishment," Advances in Production Engineering \& Management, vol. 15, no. 4, pp. 416-430, 2020.

[8] B. Wu, "Research on hospital service quality optimization decision support system based on intelligent data analysis," Electronic Design Engineering, vol. 29, no. 13, pp. 114-119, 2021.

[9] M. M. Teng, "Research on the application of intelligent financial decision support system," Science, Technology and Economy GUide, vol. 29, no. 13, pp. 55-58, 2021.

[10] Y. F. Tan and C. C. Wang, "Analysis of the effect of data mining on enterprise decision support system," Yangtze River Technology and economy, vol. 5, no. S2, pp. 203-205, 2021.

[11] Y. Wang, "Integrating system resources to build an early warning decision support platform," Gansu Science and Technology, vol. 36, no. 2, p. 78, 2007.

[12] X. Chen, "Reform of criminal procedure system under artificial intelligence technology-Taking Shanghai "206" intelligent auxiliary case handling system for criminal cases as an analysis sample," Journal of Huizhou University, vol. 39, no. 5, pp. 26-32, 2019. 
[13] S. P. Li, "Research on text classifier based on neural network algorithm," Software Guide, vol. 15, no. 23, pp. 102-103, 2007.

[14] H. Y. Li, W. Xu, Y. Cui, Z. Wang, M. Xiao, and Z. X. Sun, "Preventive maintenance decision model of urban transportation system equipment based on multi-control units," IEEE Access, vol. 8, pp. 15851-15869, 2019.

[15] X. 1. Li, J. M. Liu, and Z. Z. Shi, "Conceptual reasoning network and its application in text classification," Computer Research and Development, vol. 13, no. 9, pp. 1032-1038, 2000.

[16] Y. Fan, E. H. Chen, Q. Y. Wang, Q. S. Cai, and J. Liu, "Performance Research of hypertext coordinated classifier," Computer Research and Development, vol. 13, no. 9, pp. 1026-1031, 2000.

[17] X. J. Huang, L. D. Wu, Q. Y. Z. Shi, and G. W. Xu, "Language independent text classification method," Chinese Journal of Information, vol. 14, no. 6, pp. 1-7, 2000.

[18] Q. Diao, Y. C. Wang, H. H. Zhang, and J. He, "Word weight and classification algorithm in automatic text classification," Chinese Journal of Information, vol. 14, no. 3, pp. 25-29, 2000.

[19] D. L. Zhang, D. S. Wang, and W. M. Zheng, "Design and implementation of Chinese text classification system based on VSM," Journal of Tsinghua University, vol. 43, no. 9, pp. 1288-1291, 2003.

[20] L. S. A. A. T. Y. Thomas, "Making and validating complex decisions with the AHP/ANP," Journal of Systems Science and Systems Engineering, vol. 14, no. 1, pp. 1-36, 2005.

[21] M. Hu and S. Lingshan, "Artificial + intelligence': the basic logic of judicial intelligent reform," Zhejiang Journal, vol. 44, no. 2, pp. 12-23, 2021.

[22] T. C. Willoughby, "Review of management decision systems," in ACM SIGMIS Database, M. S. Scott Morton, Ed., vol. 4, no. 4, p. 13, Harvard University Press, Cambridge, MA, USA, 1971.

[23] W. Zeng and F. Qi, "Organization modeling based on MAS theory," Journal of Huazhong University of Technology, vol. 13, no. 8 , pp. $24-26,2000$. 